\title{
Stomporowski, Stephan
}

\section{Die Bedeutung 'Praktischen Wissens' für die Entwicklung gestaltungsorientierter Curricula}

Haushalt in Bildung \& Forschung 3 (2014) 1, S. 22-34

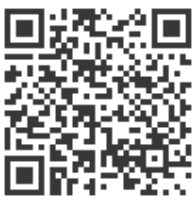

Quellenangabe/ Reference:

Stomporowski, Stephan: Die Bedeutung 'Praktischen Wissens' für die Entwicklung gestaltungsorientierter Curricula - In: Haushalt in Bildung \& Forschung 3 (2014) 1, S. 22-34 - URN: urn:nbn:de:0111-pedocs-204084 - DOI: 10.25656/01:20408

https://nbn-resolving.org/urn:nbn:de:0111-pedocs-204084

https://doi.org/10.25656/01:20408

in Kooperation mit / in cooperation with:

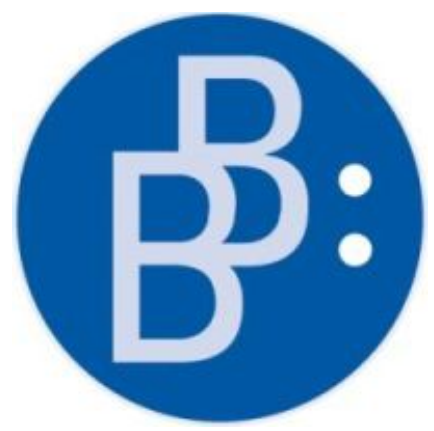

https://www.budrich.de

\section{Nutzungsbedingungen}

Gewährt wird ein nicht exklusives, nicht übertragbares, persönliches und beschränktes Recht auf Nutzung dieses Dokuments. Dieses Dokument is ausschließlich für den persönlichen, nicht-kommerziellen Gebrauch bestimmt. Die Nutzung stellt keine Übertragung des Eigentumsrechts an diesem Dokument dar und gilt vorbehaltlich der folgenden Einschränkungen Auf sämtlichen Kopien dieses Dokuments müssen alle Urheberrechtshinweise und sonstigen Hinweise auf gesetzlichen Schutz beibehalten werden. Sie dürfen dieses Dokument nicht in irgendeiner Weise abändern, noch dürfen Sie dieses Dokument für öffentliche oder kommerzielle Zwecke vervielfältigen, öffentlich ausstellen, aufführen, vertreiben oder anderweitig nutzen.

Mit der Verwendung dieses Dokuments erkennen Sie die Nutzungsbedingungen an.

\section{Kontakt / Contact:}

peDOCS

DIPF | Leibniz-Institut für Bildungsforschung und Bildungsinformation Informationszentrum (IZ) Bildung

E-Mail: pedocs@dipf.de

Internet: www.pedocs.de

\section{Terms of use}

We grant a non-exclusive, non-transferable, individual and limited right to using this document.

This document is solely intended for your personal, non-commercial use. Use of this document does not include any transfer of property rights and it is conditional to the following limitations: All of the copies of this documents must retain all copyright information and other information regarding legal protection. You are not allowed to alter this document in any way, to copy it for public or commercial purposes, to exhibit the document in public, to perform, distribute or otherwise use the document in public.

By using this particular document, you accept the above-stated conditions of use. 
3. Jahrgang Heft 1

2014

产

m

$\bullet$

$\frac{0}{2}$

$\diamond$

ธุ

\$্ণ

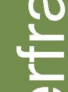

$\Phi$

$\frac{\mathrm{c}}{\mathrm{C}}$

징

인

$\stackrel{\frac{2}{亠}}{>}$

$\diamond$

$\stackrel{c}{\Phi}$

$\frac{\mathrm{O}}{\mathrm{d}}$

$\frac{\Phi}{C}$

$\bar{\Phi}$

E

E

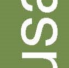

$\stackrel{\text { N }}{s}$

$\diamond$

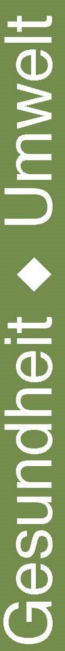

ISSN 2193-8806
Haushalt in

Forschung

\section{Schwerpunktthemen:}

Berufsdidaktische Forschung Diversität und Inklusion

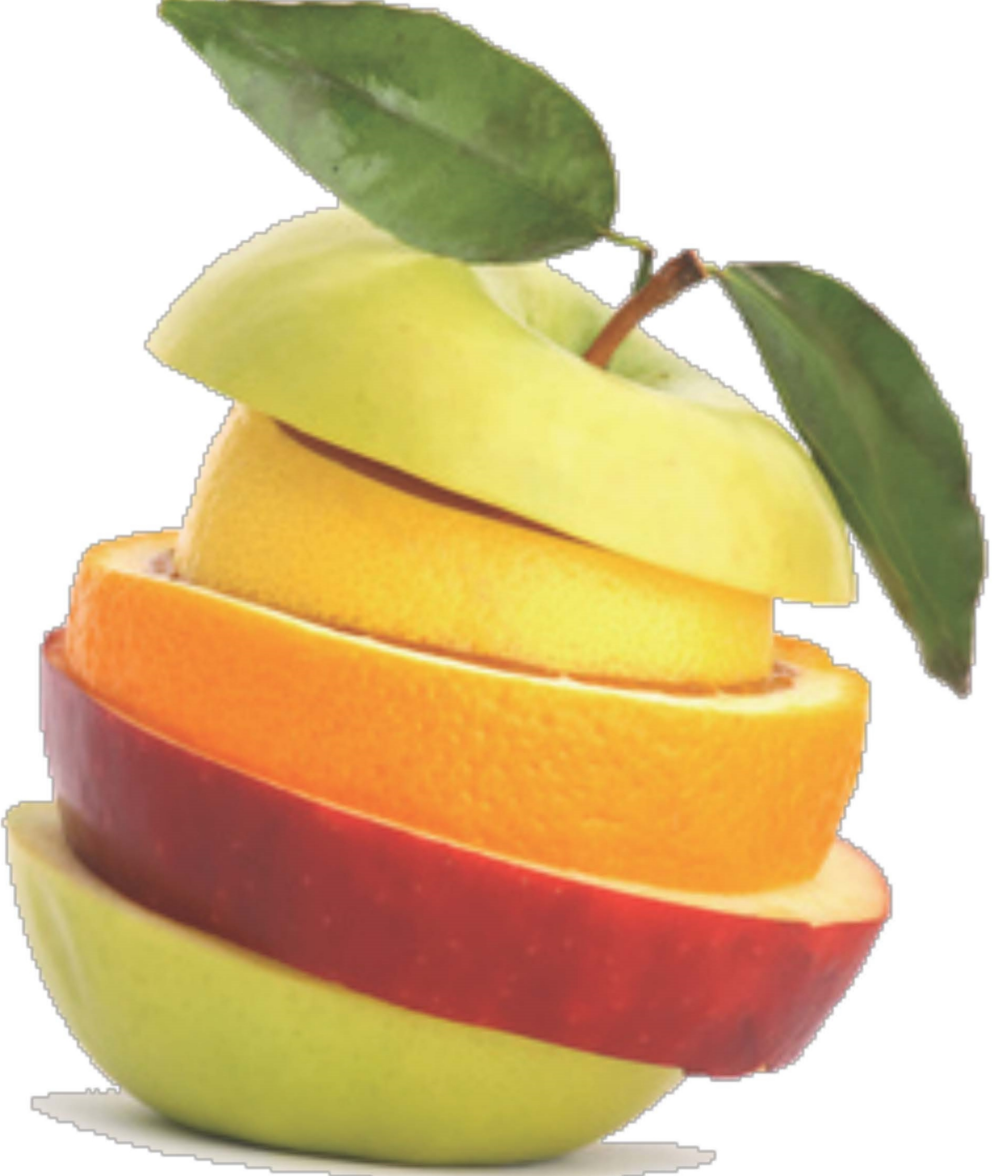

Verlag Barbara Budrich 
Inhaltsverzeichnis |

Irmhild Kettschau

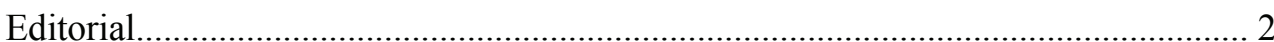

Kathrin Gemballa-Witych

Didaktisch intendierte Forschung im Berufsfeld Ernährung und Hauswirtschaft....... 3

Stephan Stomporowski

Die Bedeutung ,Praktischen Wissens` für die Entwicklung gestaltungsorientierter

Curricula

Nicole Riemer

Lernsituation „Was hat der Regenwald mit mir/mit uns zu tun?“ im Spiegel des

Angebots-Nutzungsmodells nach Helmke.

Doris Wisse

Der Konditorberuf im Spannungsfeld von handwerklicher Tradition und modernen Marktmechanismen

Josef Rützel

Inklusion als Herausforderung für die beruflichen Schulen. 61

Christine Keller und Thies Rohmann

Inklusion - Umsetzung in den Bundesländern

Mona Massumi

Diversität in der Lehrerinnen- und Lehrerbildung - zur Bedeutung von

Lehrkräften mit Migrationshintergrund.

Alexandra Brutzer

Niederschwelligkeit in personenbezogenen Dienstleistungsberufen. 96

Julia Kastrup

Rezension: Nachhaltigkeit im Berufsfeld Ernährung und Hauswirtschaft. 107

Kathrin Gemballa-Witych

Rezension: G + Einladung zum Leichenschmaus für das Gastgewerbe

Irmhild Kettschau

Bundesarbeitsgemeinschaft für Berufsbildung in der Fachrichtung Ernährung und Hauswirtschaft.

Arbeits- und Studienbuch Sensorische Prüfungen 


\title{
Stephan Stomporowski
}

\section{Die Bedeutung ,Praktischen Wissens' für die Entwicklung gestaltungsorientierter Curricula}

Gesichertes Wissen über die Relevanz beruflicher Arbeitsaufgaben, aus denen sich Praktisches Wissen generiert und über das sich gestaltungsorientierte Curricula entwickeln lassen, existiert kaum. Dabei fehlt es nicht an der Konzeption geeigneter Forschungsmethodik. Um diese nutzen zu können, muss die Bedeutung der gestaltungsorientierten Berufsbildung mit der Orientierung an dem Begriff des ,Praktischen Wissens“ in Erinnerung gerufen werden.

Schlüsselwörter: Arbeitsprozessorientierung, berufswissenschaftliche Forschung, berufliche Gestaltungskompetenz, praktisches Wissen

\section{Arbeits- und Geschäftsprozessorientierung}

Seit dem Paradigmenwechsel in der beruflichen Bildung und der Einführung des Lernfeldprinzips gilt für sämtliche Rahmenlehrpläne der verpflichtende Bezugspunkt zur Arbeits- und Geschäftsprozessorientierung:

\begin{abstract}
Arbeitsprozessorientierung im Rahmenlehrplan hat die Funktion, das Lernen an fachsystematisch strukturierten Inhalten zu überwinden zugunsten eines Lernens, dessen Inhalte auf Arbeitsprozesse bezogen sind. Hierbei sollen Arbeitsprozesse wissenschaftlich fundiert verstanden werden. Dementsprechend sollen die Lernfelder sich nicht an Teilgebieten wissenschaftlicher Fächer orientieren, sondern von Arbeitsprozessen in beruflichen Handlungsfeldern ausgehen und entsprechend strukturiert werden. (Kultusministerkonferenz (KMK), 2011, S. 29)
\end{abstract}

Grundlage jeder curricularen Arbeit stellen insofern in der beruflichen Bildung die realen Arbeitsprozesse der betrieblichen Berufspraxis dar. Aus ihnen werden die Inhalte der schulischen Berufsausbildung für den konkreten Schulunterricht generiert, die Ziele der Stoffverteilungspläne ausformuliert und das Berufsbild der Ausbildungsverordnungen praxisnah entworfen. Gleiches gilt für den Deutschen Qualifizierungsrahmen der hauswirtschaftlichen Berufe, der Lernergebnisse beschreibt, die sich explizit an der konkreten Praxis orientieren und der das erwünschte personale und fachliche Kompetenzprofil ausweist (Deutsche Gesellschaft für Hauswirtschaft e.V. (dgh), 2012, S. 11).

Damit zeichnet sich das klare Bild der Rückbindung an reale Arbeits- und Geschäftsprozesse als Grundlage der beruflichen Bildungsarbeit. Offen bleibt hingegen 


\section{Praktisches Wissen}

die Frage, was denn konkret die tatsächlich relevanten Arbeits- und Geschäftsprozesse sind, wie sich diese bestimmen lassen und inwieweit sich hierüber Kompetenzen ausbilden.

\section{Berufswissenschaftliche Forschung}

Teil der Berufsbildungsforschung ist die Berufsforschung bzw. berufswissenschaftlichen Forschung. Ihre Aufgabe besteht u.a. in der „Analyse und Systematisierung von Aufgaben, Tätigkeiten, Arbeitsmitteln" und wird auf durch Analyse berufskundlicher Literatur und über empirische Erhebungsverfahren vollzogen (Dostal, 2006, S. 107). Für den Bereich der Ernährungsberufe sowie für das Gebiet der haushaltsnahen Dienstleistungsberufe befindet sich diese Forschung jedoch ,noch am Anfang ihrer Entwicklung" (Fegebank, 2006, S. 175). Dieses Desiderat ist jedoch in den letzten Jahren in vielfacher Weise angegangen worden - zahlreiche Forschungsleistungen sind erbracht und beleuchten das Feld in eindrucksvoller Weise (z.B. im Rahmen des vom BIBB geförderten Projektes „Nachhaltigkeitsorientiertes Rahmencurriculum für die Ernährungs- und Hauswirtschaftsberufe“; vgl. Kettschau \& Mattausch 2013). Entscheidend ist, dass auf Grundlage berufswissenschaftlicher Untersuchungen detailliertes und gesichertes Wissen über die realen und relevanten Arbeits- und Geschäftsprozesse ausbuchstabiert werden kann. Ansonsten verblasst die Gestaltung von beruflichen Bildungsprozessen entweder als eine bloße Bezugnahme auf formale Richtlinien (Ausbildungsordnung, Rahmenlehrpläne) und/oder in Richtung individueller Auslegung subjektiv gewonnener eigener Erfahrungswerte. Grundlage sollte aber vielmehr das Anknüpfen an Ergebnissen der berufswissenschaftlichen Forschung in Bezug auf folgende drei Kernfragen sein (Rauner, 2005, S. 7):

(1) Welches sind die Kompetenzen, die „Fach“-Arbeiter befähigen, ihre Arbeitsaufgaben adäquat auszuführen?

(2) Über welche Fähigkeiten müssen sie darüber hinaus verfügen, um am Prozess der betrieblichen Organisationsentwicklung - im eigenen sowie über den eigenen Aufgabenbereich hinaus - mitzuwirken?

(3) Welche Fähigkeiten werden im Arbeitsprozess selbst entwickelt oder: Wie müssen lernhaltige und qualifizierende Arbeitsprozesse und Arbeitssysteme gestaltet werden?

Die formalen Ordnungsmittel repräsentieren und beantworten diese Fragen jedoch nur im Hinblick auf die betriebliche Organisationsentwicklung - nicht aber im Sinne eines sich fortlaufenden verändernden sozio-kulturellen Berufsbegriffes. Dieser interpretiert die Arbeitswirklichkeit entsprechend nicht bloß als eine empirisch gegebene und qualifikationsbezogen abzubildende, ,sondern auch als eine zu entwickelnde" sowie kritisch zu reflektierende (Rauner, 2005, S. 7). Zudem befindet sich die Arbeitswirklichkeit im stetigen Prozess ihrer Veränderung, weshalb es notwendig ist, 


\section{Praktisches Wissen}

Berufsbilder und die darauf zugeschnittenen Ordnungsmittel auf ihre Aktualität und Prospektivität hin zu untersuchen. Des Weiteren kolportiert Berufsarbeit ja nie ausschließlich fachliches Wissen und Können im Sinne von Qualifikation, sondern begründet zugleich auch Reflexionsprozesse, die einen Kompetenzgedanken markieren, der schon bei Roth mit dem Ziel der Entwicklung beruflich-moralischer Handlungsfähigkeit beschrieben wird (Roth, 1976, S. 180).

Der Begriff der Berufsform ist dabei vielfältig in der berufspädagogischen Fachliteratur entfaltet worden (z.B. Lange, 1999), wobei insbesondere Lempert seit vielen Jahren auf den elementaren Zusammenhang zwischen Qualifikation, Sozialisation und Lernen verweist (Lempert, 2006, S. 413). Berufswissenschaftliche Studien stehen daher stets im Verhältnis qualifikationsbezogener und bildungs- sowie sozialwissenschaftlich begründeter Forschung. Insofern ist die Berufsbildungsforschung Aufgabe der Berufs- und Wirtschaftspädagogik, in deren Schnittfeld die Bereiche Bildung, Sozialisation und Qualifikation stehen. Fragt man hingegen nach der institutionellen Verankerung der berufswissenschaftlichen Forschung, so kann dies nur Anliegen der domänenspezifischen Berufsfelddidaktik mit ihrem Aufgabenbereich im Schnittfeld zwischen pädagogischer und fachkundlicher Verortung sein (Fegebank, 2006, S. 174)

\section{Arbeitsprozesswissen}

Um ein Verständnis über die berufspädagogische Bedeutung des Arbeitsprozesswissens zu erhalten, muss eine grundsätzliche Annäherung an den Begriff der Handlungsregulierung erzielt werden (vgl. Pahl, Rauner \& Spöttl 2000; Rauner, 2004). Dies ist jedoch angesichts der Fülle an Literatur zum Thema Handlungsforschung und Kompetenzentwicklung durchaus kompliziert - ja sogar mit Unsicherheit verbunden, weil sich innerhalb der verschiedenen Ansätze auch erhebliche qualitative Unterschiede zeigen (dazu Jung, 2010, S. 49ff.). Vor diesem Hintergrund wird hier Position bezogen und auf den Ansatz der ,Gestaltungsorientierten Didaktik' nach Felix Rauner verwiesen.

Den Begriff der ,Gestaltungsorientierten Didaktik' verwendet Rauner erst seit einigen Jahren (z.B. Rauner, 2004; Rauner, Grollmann \& Martens, 2007). Es stellt jedoch kein konzeptionell ausformuliertes Didaktik-Modell dar, sondern ist vielmehr das Ergebnis seiner Forschung zu den Bereichen der gestaltungsorientierten Berufsbildung, des Arbeitsprozesswissens und der berufliche Kompetenzentwicklung. Insofern befindet sich der didaktische Ansatz der Gestaltungsorientierung quasi im Prozess seiner Konstituierung, besitzt aber angesichts der zahlreichen Studien, die Rauner während seiner Arbeit am Institut für Technik und Bildung (ITB) der Universität Bremen durchgeführt hat und die nun insbesondere von Seiten der TechnikDidaktik fortgeführt wird (z.B. Becker, 2013), eine fundierte und vor allem eine berufspädagogisch begründete Basis (vgl. Jung, 2010, S. 69). 


\section{Praktisches Wissen |}

Das Besondere ist dabei der von Rauner zugrunde gelegte Begriff des Arbeitsprozesswissens und seine Ausführungen zur Theorie praktischen Wissens. Hauptanliegen der von Rauner vertretenen gestaltungsorientierten Berufsbildung ist die Bedeutung empirisch gesicherten Wissens über die zentralen beruflichen Handlungssituationen, in denen sich die Auszubildenden befinden und über die sich eine Kompetenzentwicklung begründen lässt. Rauner plädiert deshalb nachhaltig für die Ausweitung berufswissenschaftlicher Studien, um empirische Gewissheit über das eigene Handlungsfeld zu erhalten. Dieser Blickwinkel begründet sich entlang folgender Überlegungen:

(1) In der empirischen Curriculumforschung zur Arbeitsprozessorientierung erhebt sich die Forderung nach einer Identifikation paradigmatischer beruflicher Handlungssituationen (Rauner, 2004, S. 4). Ziel muss es sein, das in ihnen enthaltene berufspraktische Können auf die darin enthaltenen Wissensformen auszudifferenzieren und zu explizieren, um überhaupt erst einen fundierten Anhaltspunkt für den Entwurf eines domänenspezifischen Curriculum im Kontext der beruflichen Bildung zu erhalten.

(2) Aussagen zur beruflichen Kompetenzentwicklung sind überhaupt nur dann im Sinne lerntheoretischer Begründungen als gehaltvoll anzusehen, sofern ihnen praktische Handlungssituationen zugrunde liegen, über die sich Entwicklungszuwächse im Sinne veränderter Perspektivbildung beschreiben lassen.

\subsection{Paradigmatische Arbeitsaufgaben}

Sofern die berufliche Praxis als Ausgangspunkt der Konstruktion beruflicher Bildungsprozesse anzusehen ist, stellt sich die Frage nach der Auswahl der Inhalte. In der bildungstheoretischen Didaktik besitzt die Frage nach den Auswahlkriterien den Stellenwert der grundsätzlichen Legitimation jeglichen didaktischen Handelns, zumal sich hierüber die unterrichtlichen Bildungsgehalte ausweisen lassen (Klafki, 1963, S. 126ff.; Klafki, 1996, S. 250ff.). Rauner greift in seiner gestaltungsorientierten Berufsbildung dieses prinzipielle Verständnis auf, indem er die konkrete Berufspraxis nach Merkmalen einer beruflichen Kompetenzentwicklung untersucht. Damit stellt sich die Frage nach der Bewertung der Arbeitsprozesse, also nach der Identifikation von Arbeitshandlungen, die als geeignet erscheinen, eine Kompetenzentwicklung zu befördern und damit einen curricularen Zugang zu ermöglichen. Demgegenüber greifen Ansätze der reinen Outcome-Orientierung zu kurz, weil sich hierüber kein Kriterium erschließt, das auf die Entwicklungsfähigkeit von Kompetenzen gerichtet ist. So geht es in der Berufsbildung nicht bloß um die Darstellung erwünschter Qualifikationen, sondern um die mit ihnen verbundenen Gestaltungsfähigkeiten oder anderes, um die in ihnen enthaltenen Qualitäten. Vor diesem Hintergrund verweist Rauner auf die Bedeutung der Identifizierung paradigmatischer Arbeitsaufgaben, die er wie folgt skizziert: 


\section{Praktisches Wissen}

Von charakteristischen, für die Berufsarbeit ,paradigmatischen“ Arbeitsaufgaben sprechen wir immer dann, wenn den für einen Beruf charakteristischen Arbeitszusammenhängen zugleich eine die berufliche Kompetenzentwicklung fördernde Qualität zugemessen wird. (Rauner, 2004, S. 4)

Entsprechend wird hier davon ausgegangen, dass sich in der Bewältigung beruflicher Arbeitsaufgaben zwei unterschiedliche Handlungsdimensionen widerspiegeln: qualifikationsbezogene und kompetenzbezogene Handlungsmuster. Während für die sachgerechte Ausübung von Arbeitstätigkeiten, wie das Herstellen von Speisen, die Einrichtung eines Hotelzimmers, den Empfang von Gästen usw., Qualifikationen im Sinne von Fähigkeiten, Fertigkeiten und Kenntnissen notwendig sind, führt der Kompetenzgedanke zur Bewältigung der hierin enthaltenen Problemsituationen. Diese Bewältigungshandlung setzt nun aber weniger die qualifikationsbezogene Fähigkeiten als vielmehr Aspekte des Verstehens, Reflektierens und Bewertens voraus - Eigenschaften, die im Kontext lernpsychologischer Überlegungen die Entwicklung der Persönlichkeit betreffen und insoweit den Begriff der Bildung unterstreichen (Rauner, 2008, S. 85). Vor diesem Hintergrund konturiert sich das Bild der paradigmatischen Arbeitsaufgaben als eines, das nicht nur typische berufliche Handlungssituationen abbildet, sondern zugleich auch den Aspekt der darin enthaltenen Persönlichkeitsentwicklung skizziert. Für die Bildungsarbeit erschließt sich hierüber die prinzipielle Möglichkeit zum Entwurf kontextspezifischer Fallbeschreibungen, über die eine Herleitung beruflicher Kompetenzanforderungen auf Grundlage eines entwicklungslogischen Curriculum gelingt. Dieses Curriculum basiert nun auf dem Verständnis kompetenten Handelns, welches Rauner im Modell vom ,Novizen zum Experten' entfaltet (z.B. Rauner, 2004, S. 6). Um aber dieses Bild der Generierung von entwicklungslogischen Kompetenzstufen auszudifferenzieren, ist es zunächst notwendig, den Begriff der paradigmatischen Arbeitsaufgaben an ein Verständnis der Theorie ,Praktischen Wissens' zu binden.

\subsection{Praktisches Wissen}

Ziel beruflicher Bildung ist in Anlehnung an den Kompetenzbegriff von Roth kompetentes Handeln im Sinne beruflich-moralischer Handlungsfähigkeit. In der berufswissenschaftlichen Forschung hat sich bereits in den 1980er Jahren hierfür die Bedeutung so genannten praktischen Wissens herauskristallisiert, das sich im Unterschied zum abstrakt-theoretischen Wissen für die Bewältigung beruflicher Handlungssituationen als dominant nachzeichnen und empirisch überprüfen lässt. Rauner verweist hier exemplarisch auf die Studien von Schön, der über die Erforschung von Bewältigungshandlungen in beruflichen Alltagssituationen auf die insgesamt eher rudimentäre Bedeutung abstrakt-theoretischen Wissen aufmerksam macht. Im gleichen Kontext stehen die Untersuchungen von Polany zum impliziten Wissen, welche vom österreichischen Wirtschaftspädagogen Neuweg für den deutschsprachigen Raum erschlossen wurden und eine intensive Debatte in der Berufsbildung her- 


\section{Praktisches Wissen |}

vorgerufen haben (Neuweg, 1999; Hack1, 2004). Im Kern geht es um ein Verstehen praktischen Könnens, das eben nicht primär als Ergebnis einer gezielten theoretischen Reflexionsleistung zu bewerten ist, sondern sich vor allem über Wissen generiert, das einen impliziten Charakter besitzt. So werden z.B. Probleme der ärztlichen Berufspraxis zu mehr als 80 Prozent entlang des erfahrungsbezogenen praktischen Wissens bewältigt (Rauner, 2008, S. 98). Implizites Wissen zeichnet sich danach für die Regulierung einer Bewältigungshandlung verantwortlich, kann aber selbst nicht kausallogisch expliziert und generalisiert werden. Der Grund liegt in der subjektiven Gebundenheit des impliziten Wissens, das letztlich als ein Konglomerat subjektiver Erfahrungsvorräte und subjektiver Empfindungen im Kontext der Situativität einer Handlungssituation auszuweisen ist.

$\mathrm{Zu}$ einem vergleichbaren Ergebnis kommt auch die Lehr-Lernforschung zum Thema träges Wissen. Danach erweist sich das bei Lehrerinnen und Lehrern durchaus vorhandene objektive Theoriewissen als für das Unterrichtshandeln wenig bis gar nicht handlungsregulierend. Objektiv mein hier das wissenschaftliche Wissen, das sich nicht über subjektive Erfahrungen generiert, sondern auf wissenschaftlicher Forschung beruht (Wahl, 2006, S. 29ff.). Anders die eigenen subjektiven Theorien, die sich über Jahre individuell ausgeprägt und als stabile und sichere Erkenntnisse zur Bewältigung von Handlungsproblemen ausgewiesen haben: „Das neue Wissen ,perlt ${ }^{\star}$ an den stabilen, handlungssteuernden subjektiven Theorien geringer Reichweite ab, vermag sie also nicht zu modifizieren“ (Wahl, 2006, S. 28).

Der Begriff des ,Praktischen Wissens' schließt hier prinzipiell an das implizite Wissen und an den Begriff des trägen Wissens insoweit an, als dass Rauner nach einer eindeutigen Kategorie für das Arbeitsprozesswissen fragt, deren Form sich also über die konkrete berufliche Arbeitshandlung erschließt. Allerdings zeigt sich in seiner Beschreibung des ,Praktischen Wissens' ein genereller Unterschied zum Gedanken des impliziten Wissens. Denn dieses erweist sich als „grundsätzlich nicht explizierbar, bestenfalls können Aspekte, Dimensionen, Ausrichtungen, Schwerpunkt o.ä. sprachlich re-modelliert werden ohne damit jedoch das Eigentliche des „schweigenden Wissens' zum Sprechen bringen zu können“ (Hackl, 2004, S. 109). Anders das ,Praktische Wissen“, das zwar auf implizites Wissen verweist, aber „sich zu einem großen Teil explizieren ließe, wenn die Qualifikations- und Wissensforschung ihre Forschungsmethoden verbessern würde" (Rauner, 2004, S. 23). Entscheidend, so Rauner, ist die Identifizierung und Analyse paradigmatischer Arbeitsprozesse, in denen sich berufliche Kompetenzentwicklung im Prozess reflektierter Praxiserfahrung vollzieht. Dies wiederum fordert die berufswissenschaftliche und eben im besonderen Maße die domänenspezifische Forschung heraus, die ergründeten paradigmatischen Arbeitssituationen dahingehend zu untersuchen, welches ,Praktische Wissen' in der Bewältigungshandlung zum Ausdruck kommt und wie sich dieses Kriterien geleitet auch beschreiben bzw. ausdrücken lässt. Dazu folgendes Beispiel: 


\section{Praktisches Wissen}

Ein typischer und in den Rahmenlehrplänen z.B. der Bäcker, Konditoren, Köche sowie in der Hauswirtschaft mehrfach ausgewiesener Arbeitsprozess ist die Herstellung von Teigen und Massen bzw. feiner Backwaren. Hierbei spielt Wissen über die Gärung eine zentrale Rolle, zumal sich hierüber eine Vielzahl an Erklärungen für das Gelingen und Misslingen der Backwaren, Teige usw. erschließen lässt. Wie nun genau eine Gärung in der Praxis herbeigeführt wird, das wiederum hängt von einer Reihe an Einflussfaktoren ab, deren Berücksichtigung in der Bewältigungshandlung sich im Verlauf des handwerklichen Könnens über praktische Erfahrungen ausdifferenziert und professionalisiert. Es entsteht ,Praktisches Wissen'. Insofern verweist zwar die berufliche Handlungssituation (Arbeitsprozess) auf die Herstellung von Teigen und Massen, doch die Handlungsproblematik (Bewältigungshandlung) drückt sich in der kompetenten Herbeiführung einer Gärung aus, über welche die gewünschte Eigenschaft des Produktes erzielt wird. Darin zeigt sich die Verknüpfung zwischen Qualifikation und notweniger Kompetenz, diesen Vorgang verstehen, bewerten und reflektieren zu müssen.

In diesem Beispiel zeigt sich die strukturelle Tiefe des praktischen Wissens im $\mathrm{Zu}$ sammenhang mit Gärprozessen. So lassen sich nicht nur unterschiedliche Kompetenzniveaus entfalten, sondern es kann vor allem ein spezifischer Lernbegriff ausgewiesen werden (,Gärung“), über den sich das gesamte Curriculum dimensioniert.

Es stellt sich jedoch die Frage nach der Validität des praktischen Wissensbegriffes, wie er hier mit der Bezeichnung Gärung ausgewiesen wird. Grundsätzlich ist dies Aufgabe der berufswissenschaftlichen Forschung, für die Rauner auch ein methodisches Verfahren über die Initiierung von Experten-Facharbeiter-Workshops vorschlägt (Rauner, 2005). Zu erbringen ist eine Identifizierung und Systematisierung paradigmatischer Arbeitsaufgaben, über die sich praktische Wissensbegriffe ausbuchstabieren lassen. Des Weiteren schließt Rauner an die Studien von Benner an, die über eine Vielzahl an Untersuchungen im Segment der personenbezogenen Dienstleistungsberufe den Nachweis erbracht hat, dass sich praktisches Wissen in der konkreten Berufserfahrung in sechs unterschiedlichen Dimensionen mit fortlaufender Professionalisierung immer weiter ausdifferenziert und damit auch für berufswissenschaftliche Analysen nachzeichnen lässt: Sensibilität, Kontextualität, Situativität, Paradigmatizität, Kommunikativität und Perspektivität (Rauner, 2004, S. 15ff.). Die Dimension Sensibilität meint z.B. die sich im Verlauf der Berufserfahrung herausbildende ,Fähigkeit zur Wahrnehmung und Bewertung immer feinerer und feinster Unterschiede in den berufstypischen Arbeitssituationen“" (Rauner, 2004, S. 19):

Über eine fortschreitende berufliche Kompetenz im Umgang mit Gärprozessen (Erfahrung mit unterschiedlichen Außentemperaturen und Lagerstätten, Verwendung verschiedener Mehltypen usw.) entfaltet sich entsprechend ein Mehr an praktischem Wissen, das selbst minimale Zustandsveränderungen infolge der Produkterstellung erkennen und ggf. korrigieren lässt.

Der Begriff des ,Praktischen Wissens‘ bietet damit einen gehaltvollen Anknüpfungspunkt für die berufliche Bildungsarbeit auf Grundlage paradigmatischer Arbeitsprozesse. In ihm drückt sich die Relevanz beruflicher Bewältigungshandlungen aus, die 


\section{Praktisches Wissen}

über bloßes Routinewissen und reiner fachkundiger Qualifikation hinausgeht und auf den Aspekt kompetenten Handelns aufmerksam macht. An dieser Stelle gelingt denn auch die Anbindung an ein Kompetenzmodell, das nach dem Niveau der Bewältigungshandlung fragt.

\subsection{Gestaltungskomptenz}

Kompetentes Handeln vollzieht sich nach Rauner in Entwicklungsstufen, die eine curriculare Konzeptualisierung nach dem Novizen-Experten-Modell erlaubt (siehe Abbildung 1; dazu Rauner, 1999, 2008; auch Becker, 2013).

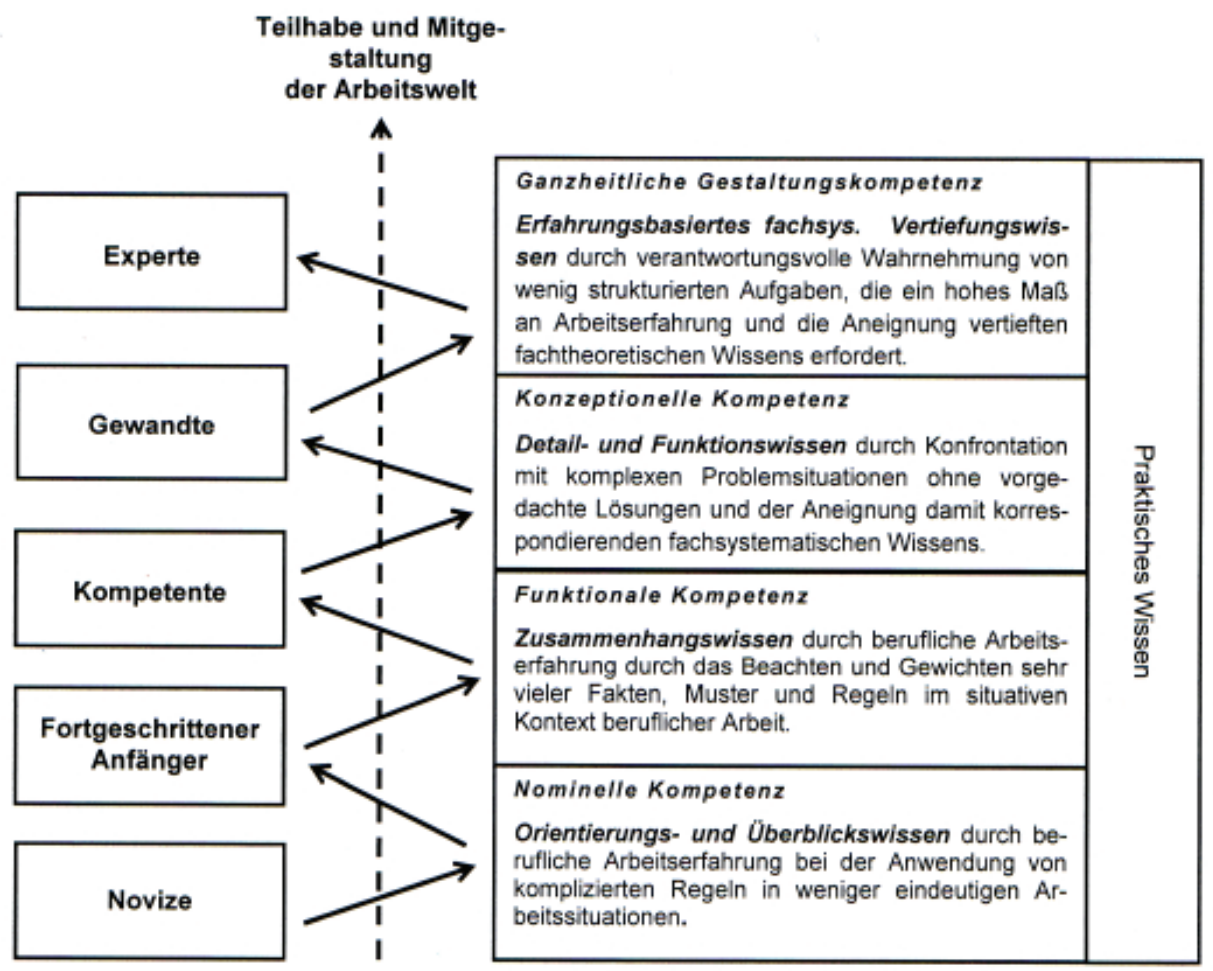

Abb. 1: Novizen-Experten-Modell (Quelle: eigene Darstellung nach Rauner)

Dieses Modell ist in der Berufs- und Wirtschaftspädagogik breit entfaltet und diskutiert worden und weist sich vor allem durch den arbeitsprozessbezogenen Bezugspunkt für didaktisches Planen aus (vgl. z.B. Pätzhold \& Rauner, 2006; Jung, 2010; Stomporowski, 2011). Jede Kompetenzstufe wird dabei auf einen spezifischen Wis- 


\section{Praktisches Wissen}

sensbereich bezogen, wodurch eine Ausdifferenzierung nach Niveaus i.S. der Literacy-Modelle gelingt.

Ein besonderes Merkmal dieses Modells ist das integrale Bildungsverständnis, das berufliche Handlungsfähigkeit mit dem Ziel verknüpft, die Arbeitswelt nicht nur zu internalisieren, um eigene Qualifikationen auszubilden, sondern an dieser über die Erweiterung der eigenen Kompetenzen aktiv teilzuhaben zum Zwecke der Gestaltung und Veränderung der Berufswirklichkeit.

In diesem Zusammenhang bricht denn auch Rauner mit dem Begriff der beruflichen Handlungskompetenz, da dieser im Unterschied zur Gestaltungskompetenz kaum die „schöpferische Qualität des selbstverantworteten Tuns sowie auf die Inhaltlichkeit der Gestaltungsspielräume verweist, die es im Prozess gesellschaftlicher Arbeit zu nutzen gilt" (Rauner, 2008, S. 93). An dieser Stelle lässt sich auf Roth verweisen, der bereits im Begriff der Kreativität ein besonderes und auszuweisendes Merkmal kompetenten Handelns (also jeglicher Sach-, Sozial- und Selbstkompetenz) sieht: „Die Geschichte der Entstehung dieser kritischen Reflexion ist die Geschichte der Entwicklung und Erziehung der menschlichen Handlungsfähigkeit zu ihrer Höchstform, wobei ,kritisch“ nur die eine Seite der Reflexion betrifft. Die andere ist ihre kreative Funktion, d.h. die Hoffnung auf die Chance, dass sich in dieser Pause Phantasie, Intuition, Imagination zu entwickeln vermögen, die zu neuen produktiven und kreativen Lösungen von Konflikten befähigen“ (Roth, 1976, S. 383).

Bezogen auf die Aspekte der paradigmatischen Arbeitsprozesse und des darin inkorporierten ,Praktischen Wissens' zeigt sich folgende Besonderheit: Jeder der von Rauner skizzierten Kompetenzstufen verweist nicht nur auf die darin enthaltenen Wissensbereiche, sondern markiert damit zugleich auch eine Wahrnehmungsperspektive. Eine Person, die z.B. komplexere Zusammenhänge beruflicher Arbeitsaufgaben versteht und Verknüpfungen zwischen den eigenen Faktenwissen und der konkreten Arbeitssituation herleiten kann (Stichwort: Zusammenhangswissen), die bewältig ihre Arbeitsaufgabe nicht nur in dieser bestimmten und kompetenten Art und Weise, sondern sie expliziert zugleich diese bestimmte berufliche Situation aus eben in jener spezifischen Kompetenz- bzw. Wahrnehmungsperspektive. Diese spezifische Wahrnehmungsperspektive beruflicher Arbeitsaufgaben wird durch den Umstand des sich allmählich herausbildenden routinemäßigen Handelns quasi fortlaufend bestätigt (die Bewältigung der Arbeitsaufgabe gelingt in immer sicherem Maße durch die wiederholende Anwendung der bewährten Lösungsstrategie). Vor diesem Hintergrund kristallisiert sich ,Praktisches Wissen“ heraus, das sich aber als eine bestimmte Form der jeweiligen Kompetenzstufe manifestiert.

Eine Veränderung dieser routinierten Handlungspraxis erfolgt dann wiederum durch neue Handlungsproblematiken, die aber von ihrer Form an ebene keine der bewährten Routinehandlungen anknüpft, quasi eine andere Perspektivwahrnehmung beinhaltet und daher zu einer Reflexion und Transformation des bisherigen Handelns auffordert. An dieser Stelle wird deutlich, dass mit der Auswahl paradigmatischer Arbeitsprozesse, die sich ebene über jene Entwicklungsmöglichkeiten auszeichnen, 


\section{Praktisches Wissen}

überhaupt erst die Chance auf eine Konstruktion eines gehaltvolles Curriculum für die beruflichen Bildungsarbeit ergibt. Gehaltvoll aus dem Grunde, weil es mit der Auswahl der im Unterricht zu behandelnden Problemstellung immer um eine Verknüpfung von Qualifikations- und Kompetenzentwicklung gehen muss - sofern mit dem Anspruch nach kompetenten Handeln zugleich auch ein Bildungsverständnis der beruflich moralischen Handlungsfähigkeit i.S. des von Roth formulierten Kompetenzansatzes ausgedrückt ist.

An dieser Stelle deckt sich das Modell des Arbeitsprozesswissens von Rauner durchaus mit der subjektwissenschaftlichen Lerntheorie von Holzkamp, der von qualitativen Lernsprüngen stets dann spricht, sofern damit ein Gewinn an subjektiver Handlungsfreiheit (Autonomie und Mündigkeit) verbunden ist (Holzkamp, 1995, $245 \mathrm{ff}$.). Anders formuliert bedeutet es für berufliche Bildungsprozesse, sofern mit der gewählten Thematik keine Möglichkeit der Erweiterung beruflicher Handlungsfreiheit subjektiv verbunden werden kann, dass Lernblockaden entstehen, welche die Kompetenzentwicklung behindern.

Zurück zum Beispiel der Gärung. Sofern zu Beginn einer Ausbildung die Auszubildenden mit der Herstellung von Teigwaren beschäftigt sind und eine Teiglockerung durch Gärung herbeiführen sollen, erfolgt i.d.R. der Einsatz z.B. von Hefe entlang einer festen Vorgabe einer Rezeptur bei Beachtung gleicher Rahmenbedingungen. Vertiefendes Detailwissen über Temperaturen, mikrobiologische Vorgänge, Standzeiten, Standorte usw. ist zu diesem Zeitpunkt der Ausbildung noch nicht vorgesehen. Es wird ein grobes Orientierungswissen vermittelt, dass am Erfolg der Bewältigungshandlung orientiert ist und den Zweck der Einübung erster Routinehandlungen der Produktherstellung unter Bedingungen beruflicher Arbeitssituationen verfolgt. Das kompetente Handeln ist auf dieser (Ausbildungs-)Stufe am Erlernen von Orientierungswissen gebunden und begründet ein erstes berufliches Selbstverständnis. Durch die Situiertheit der Arbeitsaufgabe entsteht neues praktisches Wissen und berufliche Handlungsfähigkeit. Sobald aber neue, grundsätzlich anders begründete Handlungsweisen zur Erstellung von Teigwaren im Zusammenhang mit der Gärung notwendig sind, die z.B. ein Mehr an Detailwissen notwendig machen, weil eine andere Lockerung des Produktes erwünscht ist, dann verändert sich das Wissen und formt ein neues berufliches Können. In Anlehnung an Koller kann hier von einer Transformation beruflichen Selbstverständnisses gesprochen werden (Koller, 2012, S. 144). In seiner bildungstheoretisch orientierten Biographieforschung spricht Koller von Bildung, wenn Menschen mit „Problemen konfrontiert werden, für deren Bewältigung die bisherige Figuren ihres Welt- und Selbstverhältnisses nicht mehr ausreichen" (Koller, 2012, S. 144). 


\section{Praktisches Wissen}

\section{Fazit}

Berufliche Bildung orientiert sich an Arbeits- und Geschäftsprozessen der realen beruflichen Praxis und generiert hierüber ihren pädagogischen Gegenstand. Ziel ist die Entwicklung beruflich-moralischer Handlungsfähigkeit, die auf das Mitgestalten der Arbeitswelt hinauswirkt. Für die Planung von Bildungsprozessen kommt es daher darauf an, berufliche Handlungssituationen zu identifizieren, über die eine tatsächliche Kompetenzentwicklung und nicht bloß Qualifikationsschulung gelingt. Dies setzt berufswissenschaftliche Forschung über paradigmatische Arbeitsaufgaben voraus - eine Aufgabe, die vor allem als kooperatives Feld zwischen Berufsfelddidaktik und Fachwissenschaft zu betrachten ist. Hier besteht die Aufforderung, die berufswissenschaftliche Forschung innerhalb der Domäne Ernährung und Hauswirtschaft auch weiterhin zu unterstützen und fortzuführen. Umso erfreulicher ist die Tatsache, dass sich inzwischen eine Bundesarbeitsgemeinschaft für Berufsbildung in der Fachrichtung Ernährung und Hauswirtschaft gegründet hat, um dem Entwicklungsprozess Impulse zu geben. Letztlich wird es aber Aufgabe der Vertreterinnen und Vertreter der Berufsfelddidaktik sein, hier Forschungsaktivitäten voranzutreiben.

\section{Literatur}

Becker, M. (Juli 2013). Arbeitsprozessorientierte Didaktik. [www.bwpat.de/ausgabe24/becker_bwpat24.pdf].

Deutsche Gesellschaft für Hauswirtschaft e.V. (dgh) (Hrsg.). (2012). Die hauswirtschaftlichen Berufe im Deutschen Qualifizierungsrahmen. Osnabrück.

Dostal, W. (2006). Berufsforschung. In F. Rauner (Hrsg.), Handbuch Berufsbildungsforschung (2. Aufl., S. 105-111). Bielefeld: WBV.

Erpenbeck, J. (2001). Wissensmanagement als Kompetenzmanagement. In G. Franke (Hrsg.), Komplexität und Kompetenz, ausgewählte Fragen der Kompetenzforschung (S. 102-120). Bielefeld: Bertelsmann.

Fegebank, B. (2006). Ernährung. In F. Rauner (Hrsg.), Handbuch Berufsbildungsforschung (2. Aufl., S. 170-175). Bielefeld: WBV.

Hackl, B. (2004). Explizites und implizites Wissen. Menschliches Handeln im Spannungsfeld von Intentionalität, Rationlität und praktischem Können. In B. Hackl \& G. H. Neuweg (Hrsg.), Zur Professionalisierung pädagogischen Handelns, Arbeiten aus der Sektion Lehrerbildung und Lehrerbildungsforschung in der Österreichischen Gesellschaft für Forschung und Entwicklung im Bildungswesen (S. 69-112). Münster: Lit.

Holzkamp, K. (1995). Lernen, subjektwissenschaftliche Grundlegung. Frankfurt: Campus.

Jung, E. (2010). Kompetenzerwerb, Grundlagen, Didaktik, Überprüfbarkeit. München: Oldenbourg. 


\section{Praktisches Wissen}

Kettschau, I. \& Mattausch, N. (2013). Nachhaltigkeit im Berufsfeld Ernährung und Hauswirtschaft am Beispiel der Gemeinschaftsverpflegung. Arbeitsprozesse, Qualifikationsanforderungen und Anregungen zur Umsetzung in Unterricht und Ausbildung. Hamburg: Handwerk und Technik.

Klafki, W. (1963). Studien zur Bildungstheorie und Didaktik. Weinheim: Beltz.

Klafki, W. (1996). Neue Studien zur Bildungstheorie und Didaktik, zeitgemäße Allgemeinbildung und kritisch-konstruktive Didaktik. Weinheim: Beltz.

Koller, H.-C. (2012). Bildung anders denken, Einführung in die Theorie transformatorischer Bildungsprozesse. Stuttgart: Kohlhammer.

Kultusministerkonferenz (KMK). (2011). Handreichung für die Erarbeitung von Rahmenlehrplänen der Kultusministerkonferenz für den berufbezogenen Unterricht in der Berufsschule und ihre Abstimmung mit Ausbildungsordnungen des Bundes für anerkannte Ausbildungsberufe. Berlin.

Lange, H. (1999). Die Form des Berufs. Zeitschrift für Pädagogik/Beiheft (40). 1134.

Lempert, W. (2006). Berufliche Sozialisation und berufliches Lernen. In A. Rolf (Hrsg.), Handbuch der Berufsbildung (2. Aufl., S. 413-420). Wiesbaden: Verlag für Sozialwiss.

Neuweg, G. H. (1999). Könnerschaft und implizites Wissen,zur lehrlerntheoretischen Bedeutung der Erkenntnis- und Wissenstheorie Michael Polanyis. Münster: Waxmann.

Olbrich, C. (2009). Modelle der Pflegedidaktik, mit dem Plus im Web. München: Elsevier, Urban \& Fischer.

Pahl, J.P., Rauner, F. \& Spöttl, G. (Hrsg.). (2000). Berufliches Arbeitsprozesswissen, ein Forschungsgegenstand der Berufsfeldwissenschaften. BadenBaden: Nomos-Verl.-Ges.

Pätzhold, G. \& Rauner, F. (Hrsg.). (2006). Qualifikationsforschung und Curriculumentwicklung. Zeitschrift für Berufs- und Wirtschaftspädagogik: Beihefte, 19. Stuttgart: Steiner.

Rauner, F. (1999). Entwicklungslogisch strukturierte berufliche Curricula: vom Neuling zur reflektierten Meisterschaft. Zeitschrift für Berufs- und Wirtschaftspädagogik, 95(3), 424-446.

Rauner, F. (2004). Praktisches Wissen und berufliche Handlungskompetenz. ITBForschungsberichte 14/2004. Bremen: Institut Technik und Bildung der Universität.

Rauner, F. (2005). Berufswissenschaftliche Arbeitsstudien, Zum Gegenstand und zu den Methoden derempirischen Untersuchung berufsförmig organisierter Facharbeit ITB-Arbeitspapiere Nr. 58. Bremen: Institut Technik und Bildung der Universität.

Rauner, F. (2008). Forschungen zur Kompetenzentwicklung im gewerblichtechnischen Bereich. In N. Jude, J. Hartig \& E. Klieme (Hrsg.), (Bd. 26): Kompetenzerfassung in pädagogischen Handlungsfeldern, Theorien, Konzepte 


\section{Praktisches Wissen}

und Methoden (S. 81-116). Berlin: Bundesministerium für Bildung und Forschung (BMBF).

Rauner, F., Grollmann, P. \& Martens, T. (2007). Messen beruflicher Kompetenz(entwicklung), ITB-Forschungsberichte 21/2007. Bremen: Institut Technik und Bildung der Universität.

Roth, H. (1976). Pädagogische Anthropologie (Bd. 2). Hannover: Schroedel.

Stomporowski, S. (2011). Markierungspunkte für eine Fachdidaktik Nachhaltigkeit. In S. Stomporowski (Hrsg.), Die Vitamine liegen unter der Schale, Beiträge zur Didaktik der Ernährungs- und Haushaltswissenschaften (S. 110147). Baltmannsweiler: Schneider.

Wahl, D. (2006). Lernumgebungen erfolgreich gestalten, vom trägen Wissen zum kompetenten Handeln (2. Aufl.). Bad Heilbrunn: Klinkhardt.

\section{Verfasser}

Prof. i.V. Dr. Stephan Stomporowski

Universität Hamburg

Institut für Berufs- und Wirtschaftspädagogik

Sedanstr.19

D-20146 Hamburg

E-Mail: stephan.stomporowski@uni-hamburg.de 